\title{
EL PAISAJE RURAL DE LA CAMPIÑA DE JAÉN EN LA BAJA EDAD MEDIA SEGUN LOS LIBROS DE LAS DEHESAS. Tomás Quesada Quesada
}

(Universidad de Jaén, 1994, 239 pp.)

La Historia está por escribir en muchos y variados aspectos. El desarrollo de una Historia local, aunque ha comportado serios riesgos y ha traído consigo problemas aún sin resolver, ha supuesto un nuevo e importante impulso para la investigación. Desde luego no conduce a nada si se piensa que hay que multiplicar los análisis parciales para llegar al global.

Una de las mayores posibilidades que se han abierto es el necesario cambio metodológico y la multiplicación de técnicas de investigación. Por eso, no tienen sentido las afirmaciones acerca del agotamiento de las fuentes. Se refieren, lógicamente, a las escritas, no a otras distintas. Esta discutible idea no justifica la adopción de una posición pesimista y final. Una relectura, a la luz de nuevos criterios, es fundamental. Pero ni siquiera hemos llegado a tal punto en el medievalismo, pues aún quedan muchísimos archivos por explorar y conocer. Uno de ellos, en el que se ha investigado insuficientemente, es el de la Real Chancillería de Granada. De sus fondos han ido surgiendo documentos inapreciables. Con frecuencia nos ofrecen una imagen microscópica de los espacios, hasta el punto de reconocer las tierras y los hombres. Si el impulso de la historia local fuese todo lo positivo que parece, la labor en éste y otros archivos sería continua y permanente. Hasta los últimos años no ha sido así y, en gran medida, sigue siendo vital.

Normalmente esta documentación nos ofrece una imagen que hay que confrontar con el examen del medio que describe. Un nuevo debate urge: el papel de la Arqueología, por supuesto una nueva Arqueología, en el conocimiento histórico. Es evidente que los datos arqueológicos no tienen el mismo nivel que los extraídos de las fuentes escritas. Es, sin embargo, misión del historiador, basar de forma primordial su investigación en éstas o en aquéllos, contrastarlos y, en consecuencia, producir conocimiento histórico.

En el libro que ahora publica Tomás Quesada, El paisaje rural de la campiña de Jaén en la Baja Edad Media, según los Libros de la las Dehesas, se pueden apreciar muchas de las cuestiones enunciadas. Aunque se basa en un texto del Archivo de la Real Chancillería de Granada, se observa un conocimiento de la zona que muestra el concurso de la Arqueología. Se dirá que no es tal porque sólo es la referencia a un espacio concreto y únicamente el análisis de algunos aspectos. Permítasenos que recordemos la frase de un ilustre arqueólogo medievalista francés, Jean-Marie Pesez, que resume muy bien el talante de ciertos historiadores que trabajan primordialmente con las fuentes escritas:
"Je crois qu'en outre le débat est probablement faussé par le handicap dont souffre l'archéologie, qui est la lenteur avec laquelle elle construit ses documents. Pour l'instant, il est sûr que le meilleur de l'information nous vient encore des sources écrites et de la méthode historique. Mais j’observe que I'historien ne dédaigne pas d'aller sur le terrain, voir de ses yeux les sites dont lui parle sa documentation et cela, j'y reviens et j'y insiste, ç'est déjà de la l'archéologie".

En efecto, este libro del Profesor Quesada, de la Universidad de Granada, publicado por la recentísima de Jaén, pone de relieve cómo es obligado el examen del espacio para configurar una nueva Historia. De este modo, los resultados son de un gran interés.

No es fácil resumir en esta reseña la riqueza de matices de la obra. Los textos son la base fundamental del trabajo, aunque han sido relegados a unos apéndices. Están pulcramente editados, con sus índices correspondientes bien organizados. Tienen una gran importancia y son un ejemplo de lo que aún guardan archivos tan ricos como el de la Real Chancillería de Granada. Sin embargo, Tomás Quesada ha utilizado los textos para abrir innumerables sendas. Ha de tenerse en cuenta que recoge la relación de dehesas de 1378, |40| y 1410 en torno a Jaén, en concreto a la zona al $\mathrm{N}$ de la ciudad. Se hizo un traslado en $14 \mid \mathrm{I}$, del que, a su vez, se llevó a cabo otro en 1520, que es el conservado en el Archivo de la Real Chancillería de Granada. Hay que anotar asimismo que en el origen de este segundo traslado, que es el que se edita, está el conflicto entre los intereses de los ganaderos de la ciudad, que pretenden la conservación a ultranza de las tierras de pastos, y los de los agricultores, interesados en la población y roturación de nuevas tierras.

Con cierta minuciosidad se van definiendo espacios adehesados. Tomás Quesada, con ayuda del reconocimiento territorial y, lógicamente, de la cartografía, los va delimitando e identificando en la medida que es posible. Se localizan en el valle del río Guadalbullón, especialmente en la margen izquierda, aunque también en la derecha hasta el arroyo Salado, y en la zona articulada en torno a Torredelcampo, en la Campiña giennense.

La documentación permite conocer el paisaje rural. El investigador ordena el estudio en varios apartados luego de examinar, como queda dicho, los textos y proceder a su situación. Las tierras cultivadas se organizan en espacios al $N$ de Jaén, mientras que al $S$ de la ciudad, en las tierras defendidas por las fortalezas de 
Cambil, Alhabar y Arenas, no es posible la agricultura. En las zonas más al $\mathrm{N}$ se observa, sin embargo, cómo hay reductos defensivos. Existen los denominados "castillos de la ciudad" y "un castillo de la Iglesia de Jaén", pero no son necesariamente castillos en el sentido estricto del término. Para Quesada "con esta denominación se caracteriza a aquellas fortificaciones que pertenecen a la ciudad, $u$ otro elemento del poder público como es la Iglesia y se incardinan en el entramado de defensas de la misma" (p.4I).

Gran parte de los cortijos de los que nos hablan las fuentes tienen torres. Se dibuja así un fuerte mecanismo de defensa frente a los granadinos, según el autor, aunque la historia política andaluza durante los siglo XIV y XV abunda en ejemplos de luchas de banderías.

La ciudad de Jaén se consideraba el punto principal defensivo con su fortaleza urbana. Al S los castillos de la Guardia y Pegalajar eran los fronteros. AI N, los mecanismos muestran unos asentamientos de pequeña escala protegidos por torres normalmente. Según el investigador: "Estas torres debieron ser pequeñas fortificaciones destinadas esencialmente a la defensa esporádica de los labradores y cosechas recolectadas" (p.42). Parece claro, como pone de manifiesto su estudio arqueológico que son "unas torres íntimamente ligadas al mundo agrario de la campiña giennense y muestra palpable y física de su condición de propiedad privada de sus dueños, que formaban parte de élite de la ciudad y concejo de Jaén" (p.43). Esta ocupación del territorio se intensifica tanto por una extensión de las áreas de cultivo puestas ya en funcionamiento, como por la apertura de otras nuevas. En contraste, la zona $S$ de Jaén, era un auténtico desierto humano antes de 1492. Empieza a romperse tras la caída del reino granadino. En 1508 , la reina $D^{a}$ Juana concede licencia al concejo de Jaén para repoblar el área meridional de la jurisdicción, pero con ciertas salvaguardas, en atención a los intereses ganaderos de la ciudad. No obstante, el proceso repoblador, sería seriamente dificultado y no se llevaría a cabo hasta fechas más tardías, bien entrado el siglo XVI.

De esta manera, en el capítulo dedicado al área de cultivo y su evolución, Tomás Quesada dibuja un rápido panorama sobre la organización del territorio, aunque no se examinen las relaciones jurídicas entre la ciudad y su distrito. Por eso, quedan algunas cuestiones en el aire que, sin duda, se debieran resolver en los próximos trabajos, en otros que no tengan el carácter de éste, pionero y basado primordialmente en una serie de textos. De entre otros aspectos, cabría plantear, sobre todo para el interés de quien hace esta reseña, algunos de especial incidencia en el poblamiento. Es difícil con una documentación muy posterior a la conquista castellana de la zona, poder establecer unas líneas básicas elementales. Así, según creemos, es necesario saber la cronología de los castillos que se mencionan. Tal vez proceden de época árabe, quizás almohade, pero nos falta la constatación. Otro tema muy interesante es conocer el origen de los cortijos. Pueden ser fundaciones anteriores e incluso con un carácter distinto; tal vez se traten de alquerías andalusíes ya abandonadas, ya ocupadas mínimamente. Las torres y el estudio arqueológico en profundidad pueden ofrecer un volumen de datos importante al respecto. Hasta ahora los informes con que contamos (p. 42, nota 64) no dejan bien establecido el problema. Podrían tratarse asimismo de asentamientos nuevos y no permanentes, conectados con la nueva élite giennense. Pero tanto en su caso como en otro, se deben plantear sin dudas unas perspectivas de relación con la ciudad y con respecto a todo el territorio.

Algunos aspectos de tales características vuelven a surgir en el siguiente capítulo, el dedicado al paisaje rural. Aunque la delimitación de las dehesas para ganado de labor no permite conocer las ocupaciones agrícolas, hay indicios suficientes para plantear ciertas cuestiones. El cultivo por excelencia era el cereal, posiblemente el trigo. Otros aparecen citados de forma esporádica: la vid, principalmente, pero también árboles como almendros, olivos y morales. Es más, se observa, al contrario de lo que ocurre con el cereal, una concentración de estas especies vegetales.

Un problema surge de manera inmediata. Se trata de la intensidad de los cultivos y, como lógica derivación, la existencia y extensión del regadío. A este respecto, la documentación utilizada sólo refiere "huelgas" o pequeñas parcelas irrigadas en las inmediaciones de los cursos de agua, sin que se citen "huertas". Aparecen no obstante, elementos propios del regadío, como acequias, pozos y fuentes. Se dibuja un panorama en el que la agricultura de regadío es escasa, mientras que la vida ganadera es importante. Es más, el propio autor indica con respecto a las obras hidráulicas que muy esporádicamente aparecen citadas, que se utilizaban primordialmente como fuerza motriz y de forma subsidiaria para regar. Y añade :'La propia ubicación de estos cortijos en la inmediaciones del río Guadalbullón, en una cota bastante baja, ya nos ponen (sic) en guardia, en este mismo sentido, de la poca cantidad de tierra que pudieron regar, atendiendo al poco desnivel entre la acequia y el río" (p.6I).

Si la vida agrícola y las especies cultivadas apenas se pueden conocer por los libros de las dehesas, no es igual para la vegetación natural. A través de la identificación de 22 plantas, el autor puede manifestar la existencia de tres paisajes distintos. En primer lugar, se percibe un bosque galería con árboles de ribera, como el álamo y el sauce. Un segundo tipo de vegetación es el propio de tierras salinas, ya que hay cursos de agua salada en la campiña. Finalmente, el bosque mediterráneo, en distintas fases subseriales, pero apareciendo árboles como la encina y el quejigo y otras plantas propias del matorral (cambrones, gamones y retamas entre otros).

Aún cuando es difícil sacar conclusiones, el prof. Quesada plantea una serie de cuestiones importantes acerca de la vida agrícola y el paisaje rural. Sin duda tendrá que contarse con ellas cuando se investiguen otras zonas y éstas de la campiña de Jaén. 
Las afirmaciones, a veces arriesgadas, eran necesarias. Primero dice: "Y en este sentido la presencia de plantas propias de un grado de degradación del encinar tiene una especial significación, ya que son testigos de una época en la que un uso del suelo más intensivo obligó a la tala de los encinares. Esta imagen que nos da la vegetación de una campiña que conoció un período en que se explotaba con mayor intensidad se ve reforzada con los datos que hemos analizado más arriba referidos a la presencia inconexa de elementos de un área irrigada amplia. Todo parece indicar que en una etapa anterior, probablemente antes de la conquista de la ciudad por los castellanos en 1246, la campiña de Jaén conoció una organización agrícola mucho más intensa. Esta debió de verse afectada durante el siglo XIII por las campañas realizadas por las tropas de Fernando III en la campiña giennense..." (p. 73).

A nivel de hipótesis son muy sugestivas tales afirmaciones, pero es preciso poner de manifiesto que la vegetación debe examinarse con mucha atención. En primer lugar, la presencia de una especie vegetal es sólo un indicio, porque, a veces, se pone de manifiesto precisamente por su singularidad. Pero también una planta debe situarse en el medio edáfico en que crece, por lo que no significa siempre un estado de degradación.

De todas formas, en el marco de este libro, el capitulo en cuestión es de una gran calidad y se pueden suscribir, aunque sea como punto de partida, las palabras con que se cierra: "Como consecuencia de estas campañas la organización agrícola de la campiña debió de sufrir un grave quebranto, siendo finalmente abandonada cuando los nuevos pobladores cristianos reordenaron su espacio hacia otro tipo de agricultura, utilizando sólo las tierras más fértiles y aquellos elementos que les fueron de utilidad, disgregando el conjunto de acequias y huertas anteriores. Fruto de esta transformación sería la aparición de una vegetación de retamales, gamonales, atochares sobre los terrenos abandonados" (p. 74).

A partir de aquí es cuando cobra especial significación el capítulo siguiente, acerca de los despoblados y otros testimonios del pasado.

Un primer indicio de ocupación y organización de la campiña es la existencia de "una caminería capilar que atraviesa en todas direcciones la campiña"(p.78). Pero de mayor importancia son, sin embargo, los despoblados. Son II los que se citan en la parte $\mathrm{N}$ de Jaén, aunque no se pone de manifiesto ninguna característica sobre ellos ni mucho menos se puede precisar cuándo se abandonan. Uno de ellos es el yacimiento excavado de Peñaflor. Es indudable que el trabajo arqueológico tiene todavía mucho que decir a este respecto de los despoblados. Se precisa pues, marcar los ritmos de ocupación y abandono, sin detener la investigación en la conquista castellana en línea con algunos intentos que ya se han hecho.

Una última parte es la dedicada al estudio de las salinas, tema que ocupa ahora de manera muy intensa e inteligente al autor de esta obra. Sólo es un inicio de lo que investiga Tomás Quesada en la actualidad y como tal debe considerarse. Cierra su libro, antes de los anejos ya mencionados, con este párrafo que resume su trabajo: "En definitiva, los diferentes elementos que definen el paisaje de la campiña de Jaén durante los siglos XIV y XV nos llevan a la conclusión de que ésta se encontraba en un período de explotación atenuada, en un proceso de transformación desde un ordenamiento del espacio creado en época islámica que es abandonado tras la conquista cristiana del siglo XIII y que paulatinamente va siendo sustituido por otro creado por los castellanos conforme se afianza su dominio del territorio que no llega a ser completo hasta bien entrado el siglo XV, cuando de nuevo los recursos agrícolas y naturales de la campiña vuelvan a ser explotados con una mayor intensidad"' (pp. 92-93).

Antes de terminar esta amplia reseña no queremos dejar de decir algunas cosas. Ante todo señalar que este libro pone de manifiesto, gracias al buen hacer de Tomás Quesada, las posibilidades en la investigación histórica. El análisis de las fuentes escritas y de la arqueología han de servir a un mejor conocimiento histórico. Aunque queda mucho camino por recorrer, hay que saludar obras como ésta. Su autor ya ha mostrado que es necesario ir por un camino difícil, pero obligado. Por último, debemos manifestar el buen criterio del Servicio de Publicaciones e Intercambio científico de la Universidad de Jaén, que ha hecho posible un catálogo bien seleccionado y de indudable calidad en el que incluye el presente libro.

Antonio Malpica Cuello 